\title{
The Effect of Oxytetracycline on Leukocyte Migration into Inflammatory Exudate
}

The migration of leukocytes to sites of infection and tissue injury is a primary defence mechanism. When localized at the infection site polymorphonuclear leucocytes phagocytose bacteria and other foreign particles. The leukocyte is also of interest as a source of inflammation mediators e.g. metabolites of arachidonic acid (Simmons et al. 1983).

Functioning leucocytes are of vital importance for the succesful outcome of antibacterial therapy. The ideal situation is achieved when the host's defence mechanisms and the antibacterial drug act synergistically. The relationship between the host and the invading bacteria is very complex and may be further complicated by the fact that certain antibacterial drugs may impair some of the non-specific defence mechanisms.

The literature on the effects of antibacterial drugs on leukocyte function has grown rapidly during the last decade. Several comprehensive reviews have been published on this topic (e.g. Milatovic 1983). Tetracyclines is a group of drugs which has often been reported to impair leukocyte migration in vitro.

As oxytetracycline (OTC) is widely used in veterinary medicine it was considered to be of interest to study its effect on leukocyte migration in vivo.

Several techniques have been used to study leukocyte migration in vivo. The most used method includes induction of an inflammatory process in the peritoneal or pleural cavities of small laboratory animals. Samples can easily be obtained from these cavities for cell counting and the study of cell function (Bird \& Girod 1985). Most of the basic knowledge about leukocyte migration in vivo is based on results from studies using these or similar methods.

Tissue cages were used for the study of leukocyte migration in the present experiments. The tissue cage is a partly perforated capsule of an inert material which can be implanted e.g. subcutaneously. After 2-3 weeks there is an ingrowth of granulation tissue through the perforations, leaving a fluid-filled cavity in the middle. The tissue cage technique was originally developed for sampling of interstitial fluid and has been widely used in pharmacokinetic studies, but also in studies of local inflammatory reactions. The technique is e.g. excellent for the study of local prostaglandin release (Higgins et al. 1984, Luthman et al. 1988).

In the present study 6 calves were used in a cross-over experiment. All animals carried 4 subcutaneously implanted tissue cages of the type described by Bengtsson et al. (1984). The cages had been in situ for 5 weeks and had been previously used in pharmacokinetic studies. An inflammatory reaction was induced within a cage by injection of $0.5 \mathrm{ml}$ of a $1 \%$ carragenan solution. Three of the animals were given an intravenous injection of OTC $(10 \mathrm{mg} / \mathrm{kg})$ immediately before the carragenan injection. The OTC injection was repeated after $12 \mathrm{~h}$. The remaining 3 animals served as controls. The experiment was repeated after 1 week in a way so that all animals had been treated with OTC and 


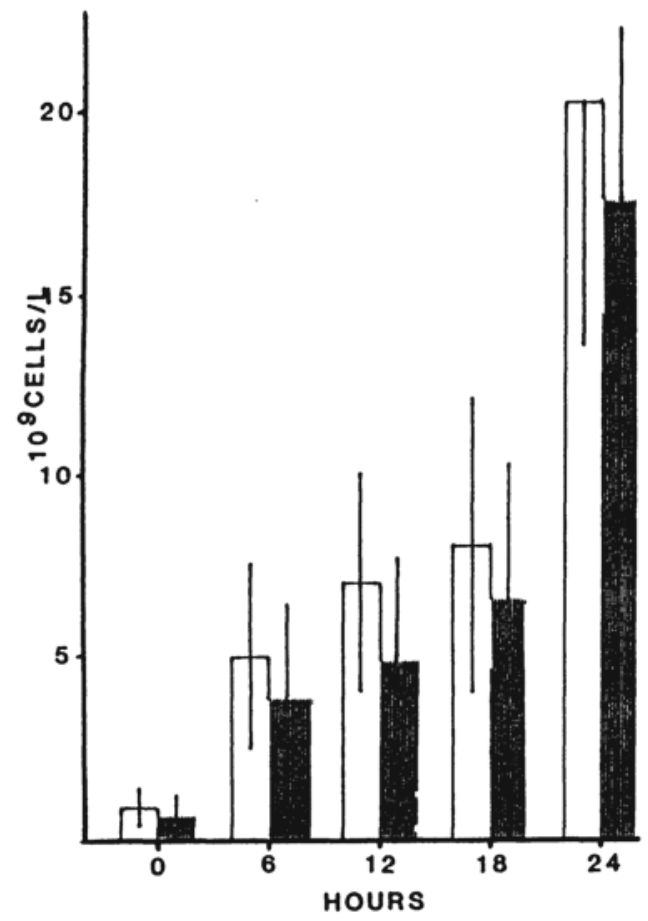

Figure 1. Changes in the number of leukocytes in tissue cage fluid after injection of carragenan into the cage, before (open bars) and after (shadowed bars) oxytetracycline treatment.

$$
\mathrm{n}=6, \mathrm{X} \pm \text { SEM. }
$$

served as controls at the end of the study. Different cages were used in each part of the experiment.

OTC was obtained from Sigma Biochemicals and was dissolved in saline immediately before use.

Tissue cage fluid (TCF) was sampled as described by Bengtsson et al. (1984) for counting of leukocytes. It was found necessary to dilute TCF in an equal volume of Hank's solution containing $0.5 \%$ trypsin to avoid aggregation of the cells.

All injected cages responded with a significant increase in the number of leukocytes in TCF. The highest number was recorded after 24 h. As shown in Fig. 1 repeated OTC treatment did not significantly alter leukocyte migration into TCF.

The serum concentration-time profile of OTC was very similar to what was earlier reported in calves after the same treatment (Bengtsson et al. 1986).

The results showed that tissue cages can be used for the study of leukocyte migration in cattle. There was however a very wide variation in respons to carragenan between individual cages. It is possible that this variation can be reduced by using a different cage model or a more concentrated carragenan solution.

The clinical implications of in vitro studies showing an inhibitory effect of antibacterial drugs on leukocyte function is difficult to evaluate. From a theoretical point of view it should be doubtful to use drugs which seriously impair the non-specific defence mechanisms when the antibiogram of the invading bacteria is unknown. The results presented in Fig. 1 do not support the findings from most in vitro studies.

Jan Luthman, Björn Bengtsson and Christer Korpe.

Department of Cattle and Sheep Diseases, Faculty of Veterinary Medicine,

Swedish University of Agricultural Sciences, Uppsala.

\section{References}

Bengtsson B, Luthman J, Jacobsson SO: Evaluation of a tissue cage model for use in cattle. Acta vet. scand. 1984, 25, 480-494.

Bengtsson B, Luthman J. Jacobsson SO: The penetration of oxytetracycline into tissue cage fluid in calves. J. vet. Pharmacol. Therap. 1986, 9, 71-80.

Bird J, Girod JP: An appraisal of the technique of polymorphonuclear leukocyte chemiluminescence as a method to detect compounds with anti-inflammatory activity. J. Pharmacol. Methods, 1985, 14, 305-312. 
Higgins $J$, Lees $P$, Wright JA: Tissue cage model for the collection of inflammatory exudate in ponies. Res. Vet. Sci. 1984, 36, 284-289.

Luthman J, Kindahl H, Jacobsson SO, Thunberg $L$ : Local and general effects of Salmonella typhimurium endotocin in calves. J. vet. Med. A, 1988, 35, 586-595.
Milatovic D: Antibiotics and phagocytosis. Eur. J. clin. Microbiol. 1983, 2, 414-425.

Simmons PM, Salmon JA, Moncada S: The release of leukotriene B during experimental inflammation. Pharmacol. 1983, 32, 13531359.

(Received October 17, 1988)

Reprints may be requested from: Jan Luthman, Department of Cattle and Sheep Diseases, Swedish University of Agricultural Sciences, S-750 07 Uppsala, Sweden. 
